\title{
Segmented scintillators with SiPM readout for measuring antiproton annihilations
}

\author{
Anna Sótér* ${ }^{*}$ Daniel Barna, ${ }^{b c}$ Koichi Todoroki, ${ }^{d}$ Takumi Kobayashi, ${ }^{d}$ \\ Dezsö Horváth, ${ }^{c}$ Masaki Hori ${ }^{a d}$ \\ ${ }^{a}$ Max-Planck-Institut für Quantenoptik, D-85748 Garching, Germany \\ ${ }^{b}$ CERN CH-1211 Geneva 23, Switzerland \\ ${ }^{c}$ Wigner Research Center for Physics, H-1525 Budapest, Hungary \\ ${ }^{d}$ Department of Physics, University of Tokyo, Tokyo 113-0033, Japan
}

The Atomic Spectroscopy and Collisions Using Slow Antiprotons (ASACUSA) experiment at CERN constructed some segmented scintillation counters to measure and track the charged pions emerging from antiproton annihilations in a future superconducting radiofrequency Paul trap for antiprotons. The photoelectron yields of various scintillator configurations were measured using a $\pi^{-}$beam of momentum $p \approx 1 \mathrm{GeV} / \mathrm{c}$ at the T9 beamline of the CERN Proton Synchrotron (PS). Various fibers and silicon photomultipliers, fiber end terminations, and couplings between the fibers and scintillators were compared. The detectors were also tested using the antiproton beam of the Antiproton Decelerator (AD). At high antiproton rates a saturation was observed in the scintillator signal. We review the detector construction and measurement results.

Technology and Instrumentation in Particle Physics 2014,

2-6 June, 2014

Amsterdam, the Netherlands

\footnotetext{
*Speaker.
} 


\section{Detector construction}

The ASACUSA experiment recently developed segmented scintillators with silicon photomultiplier (SiPM) readout [1] to measure and track the charged pions which emerge from antiproton annihilations [2, 3, 4] that occur in a superconducting Paul trap (see Fig. 1). The detectors were also used in a recent experiment which attempted to measure the cross-sections of antiproton annihilations on various target foils [5]. The detectors consisted of 541 scintillator bars with a spatial resolution of $\sim 17 \mathrm{~mm}$. They were arranged in 11 detector modules around the trap.

Seven modules [Fig. 2 (b)] had sensitive areas between $0.25 \times 0.45 \mathrm{~m}^{2}$ and $0.8 \times 0.45 \mathrm{~m}^{2}$, and contained cast scintillator bars (Saint-Gobain Crystals BC-408) with a cross section of $50 \times 12.7$ $\mathrm{mm}^{2}$ arranged in a XY geometry. Four modules [Fig. 2 (a)] had active areas $\sim 1 \times 1 \mathrm{~m}^{2}$, and contained extruded scintillators developed in Fermilab [6, 7], with a cross section of $17 \times 19 \mathrm{~mm}^{2}$. The latter modules were designed for higher spatial resolution and tracking efficiency of the pions $(>15 \%)$.

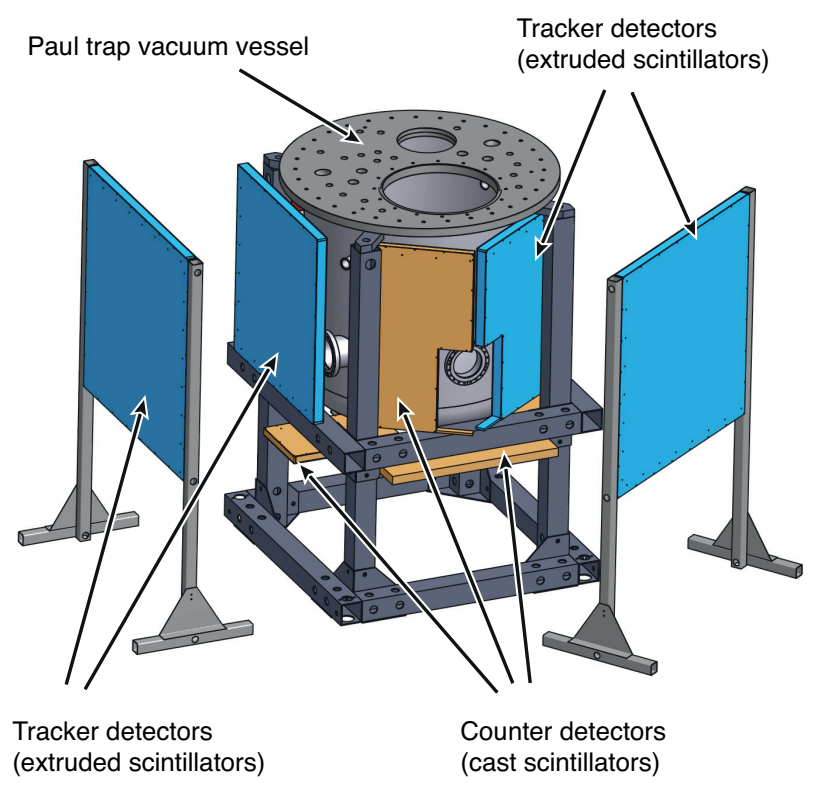

Figure 1: Diagram of the scintillation detector modules surrounding the future Paul trap for antiprotons of the ASACUSA collaboration. It contains two pairs of modules (blue) consisting of $17 \mathrm{~mm}$-wide extruded scintillator bars, and seven additional modules (orange) containing cast scintillators. Two of the blue tracker modules were already used in different setup in ASACUSA [5] to measure the cross-secton of slow $\bar{p}$ annihilating in-flight. Figure from Ref. [1].

The detectors were housed in aluminum boxes that were inlayed with black sheets (Fig. 2). Green wavelength shifting (WLS) fibers [Kuraray Co., Ltd. Y-11(200)M)] of diameter $d=1.2$ mm embedded in each bar collected the scintillation light. The light was coupled to a SiPM with a sensitive area of $1 \times 1 \mathrm{~mm}^{2}$ (Hamamatsu MPPC type S10362-11-025C, [8, 9]) using a plastic connector [10]. Each SiPM contained 1600 pixels.

\section{Measurement results}

The photoelectron yield $\Gamma$ of the scintillators were measured using the $1-\mathrm{GeV} / \mathrm{c}$ momentum pion beam using the T9 beamline of CERN. The $\Gamma$-values were corrected for spurious crosstalk and afterpulsing effects. The cast scintillators of thickness $t_{d}=12.7 \mathrm{~mm}$ yielded values of $\Gamma=35-39$, while with the Fermilab extruded scintillators of $19 \times 17 \mathrm{~mm}^{2}$ cross section yielded $34-38$. The 

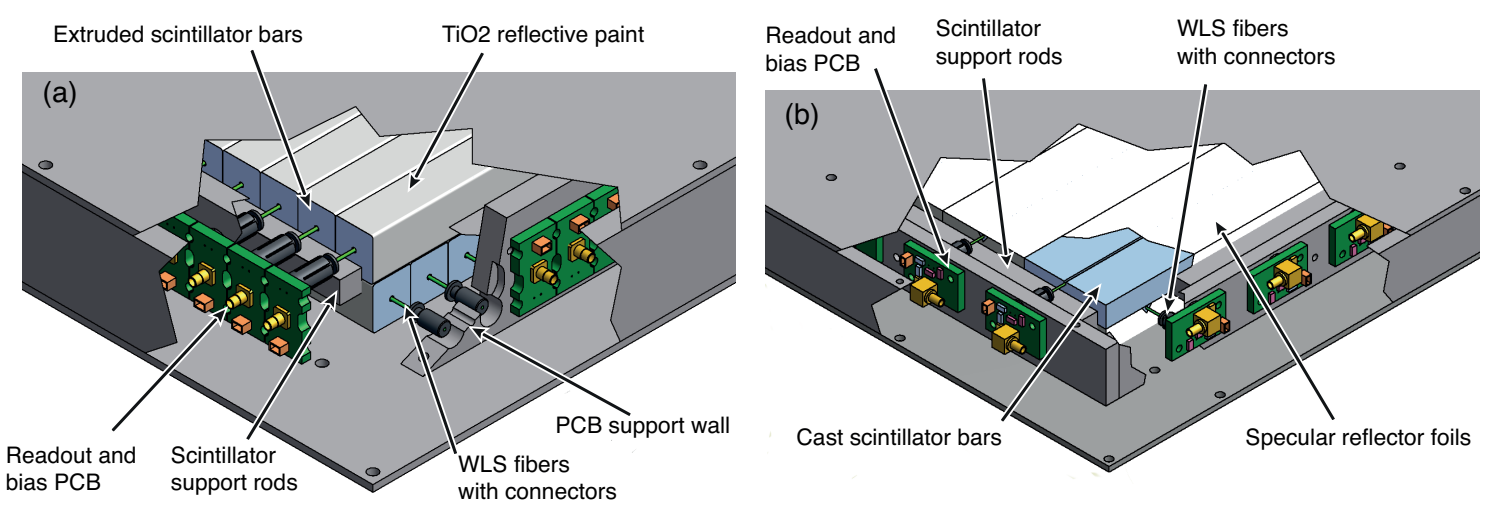

Figure 2: (a) Layout of the extruded scintillator module. A green WLS fiber was placed into a hole fabricated in the center of each scintillator bar. (b) Layout of the cast scintillator module, with fibers embedded into machined U-shaped grooves on the surface of the scintillator bars. The fibers were coupled to SiPMs of size $1 \mathrm{~mm} \times 1 \mathrm{~mm}$. Figures from Ref.[1].

yields of the extruded bars were $\sim 30 \%$ less than those of cast scintillators of the same thickness, which roughly agrees with the results reported by other groups [11, 12, 13]. Both scintillators provide sufficient single-to-noise ratio $(>6)$ above the dark current for detecting minimum-ionizing particles. The spatial uniformity of the cast scintillators over the 1-m-long bar was typically within $10 \%$, whereas for extruded ones it was $<30 \%$. More details can be found in Ref. [1].

The response of the scintillators against antiproton annihilations was also measured. For this we used a 300-ns long pulsed beam containing $(6-7) \times 10^{6}$ antiprotons with a kinetic energy $E=70 \mathrm{keV}[14,15]$ provided by the AD and radiofrequency quadrupole decelerator (RFQD). A Cherenkov detector [2] made of UV-transparent acrylic glass measured the flux of pions that emerged from the antiproton annihilations. It was read out by a fine-mesh photomultiplier (Hamamatsu R5505GX-ASSYII). In Fig. 3 (a), the waveform of the signal taken as an average of 14 antiproton pulses is shown. The instantaneous flux of pions was so high that individual annihilations could not be resolved. The 300-ns-long Gaussian-shaped signal at $t \sim 2250 \mathrm{~ns}$ corresponds to the envelope of the pulsed beam of antiprotons.

We placed a cast scintillator of size $750 \times 50 \times 10 \mathrm{~mm}^{3}$ with SiPM readout at the same position. In Fig. 3 (b), the signal measured at a bias voltage $\sim 4 \mathrm{~V}$ below the Geiger breakdown threshold $V_{0}$ is shown. The SiPM operated as an avalanche photodiode of low gain. A single peak of amplitude $\sim 2 \mathrm{mV}$ appeared at $t \sim 2250 \mathrm{~ns}$ as in the Cherenkov counter [Fig. 3 (a)].

As $V_{\text {bias }}$ neared the breakdown voltage [Fig. 3 (c)], two secondary peaks at $t \sim 950$ and 1800 ns appeared, while the signal amplitude increased by an order of magnitude, to $\sim 20 \mathrm{mV}$. This was caused by the higher sensitivity and gain of the SiPM, which now detected the weak scintillation light that arose from the annihilations that occurred elsewhere in the experimental beamline. More structures appeared at a bias $V_{\text {bias }}=69 \mathrm{~V}$ [Fig. 3 (d)]. Between $V_{\text {bias }}=72$ and $73.5 \mathrm{~V}$ [Fig. 3 (e)(f)], the amplitude of the peak at $t=2250 \mathrm{~ns}$ became saturated, and the waveform was deformed by the recharging time of the bias circuit and SiPM. We estimate that at a typical bias of $V_{\text {bias }} \sim$ $\left(V_{0}+1.5\right) \sim 70.5 \mathrm{~V}, \sim 10$ simultaneous pion hits on the scintillator would saturate the SiPMs.

The amplitudes of the two intense peaks at $t=950 \mathrm{~ns}$ (filled triangles) and $2250 \mathrm{~ns}$ (circles) 

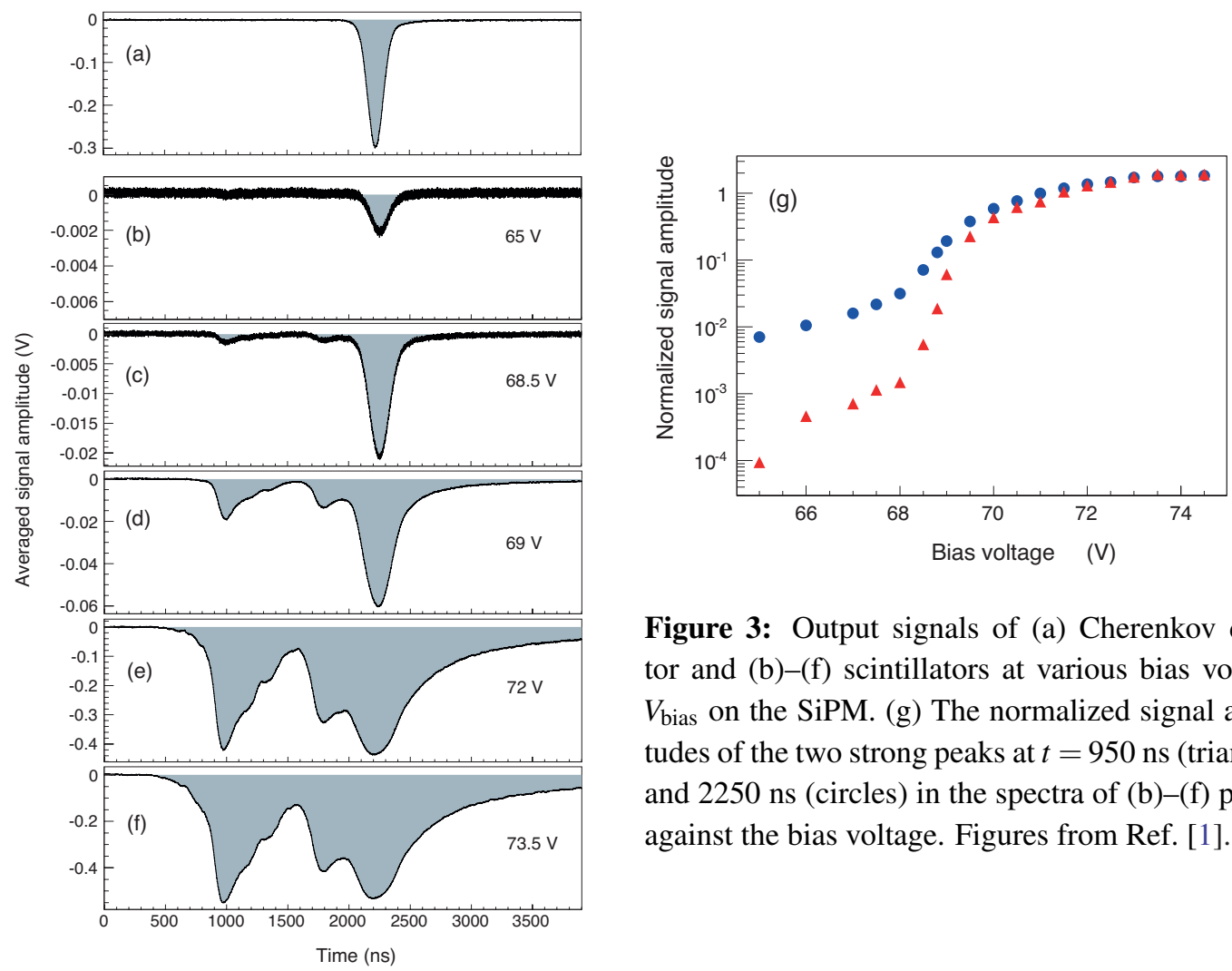

Figure 3: Output signals of (a) Cherenkov detector and (b)-(f) scintillators at various bias voltages $V_{\text {bias }}$ on the SiPM. (g) The normalized signal amplitudes of the two strong peaks at $t=950 \mathrm{~ns}$ (triangles) and $2250 \mathrm{~ns}$ (circles) in the spectra of (b)-(f) plotted against the bias voltage. Figures from Ref. [1].

normalized to the antiproton beam intensity are shown in Fig. $3(\mathrm{~g})$ as a function of $V_{\text {bias }}$. The rapid increase at $\sim 68.8 \mathrm{~V}$ corresponds to the Geiger breakdown threshold.

These results showed that both operation modes of the detectors can be exploited in our experiments; at a low bias ( $V_{\text {bias }} \sim\left(V_{0}-4\right) \sim 65 \mathrm{~V}$ ) the timing profile of the antiproton beam can be measured with high dynamic range and timing resolution, and operating them above the Geigerthreshold $\left(V_{\text {bias }} \sim\left(V_{0}+1.5\right) \sim 70.5 \mathrm{~V}\right)$ allows us to detect single pion hits with high efficiency and linear response up to $\sim 10$ simultaneous hits.

\section{Acknowledgments}

We are deeply indebted to A. Pla-Dalmau for supplying the extruded scintillators used in this work and for fruitful discussions. We thank the ASACUSA collaboration, R. Dumps, L. Gatignon, A. László, A. Minamino, T. Nakaya, K. Niita, T. Schneider, and P. Zalán. This work was supported by the European Research Council (ERC-StG), and the Hungarian Research Foundation (K103917).

\section{References}

[1] A. Sótér et al., Rev. Sci. Instrum. 85, 023302 (2014).

[2] M. Hori et al., Nucl. Instrum. Methods Phys. Res. A 496, 102 (2003).

[3] M. Hori et al., Phys. Rev. Lett. 87, 093401 (2001). 
[4] M. Hori et al., Nature 475, 484 (2011).

[5] H. Aghai-Khozani et al., Eur. Phys. J. Plus 127, 125 (2012).

[6] A. Pla-Dalmau, A. D. Bross, and K. L. Mellott, Nucl. Instrum. Methods Phys. Res. A 466, 482 (2001).

[7] A. Pla-Dalmau, A. D. Bross, and V. V. Rykalin, in IEEE Nuclear Science Symposium Conference Record, Portland, 2003, (IEEE, Piscataway, NJ, 2003) pp. 102-104.

[8] K. Yamamoto et al., in IEEE Nuclear Science Symposium Conference Record, San Diego, 2006 (IEEE, Piscataway, NJ, 2006) pp. 1094-1097.

[9] S. Gomi et al., Nucl. Instrum. Methods Phys. Res. A 581, 427 (2007).

[10] H. Kawamuko et al., PoS PD07, 043 (2007).

[11] A. Pla-Dalmau, A. D. Bross, and K. L. Mellott, arXiv preprint physics/9904004 (1999).

[12] A. Izmaylov et al., Nucl. Instrum. Methods Phys. Res. A 623, 382 (2010).

[13] V. Balagura et al., Nucl. Instrum. Methods Phys. Res. A 564, 590 (2006)

[14] M. Hori et al., Phys. Rev. Lett. 91, 123401 (2003).

[15] M. Hori et al., Phys. Rev. Lett. 96, 243401 (2006). 